\title{
Pulmonary Sarcoidosis Concurrent with Lupus Pernio and Peri-aortitis
}

\author{
Kyawzaw Lin ${ }^{1}$, Sithu Lin ${ }^{1}$, Aung Naing Lin ${ }^{1}$, Thinzar Lin ${ }^{1}$, Louis Gerolemou ${ }^{2}$, Ameer Rasheed ${ }^{2}$ \\ Departments of ${ }^{1}$ Internal Medicine and ${ }^{2}$ Pulmonology, The Brooklyn Hospital Center, Affiliate of the Mount Sinai Hospital, \\ 121, Dekalb Avenue, Brooklyn, NY 11201.
}

\section{Corresponding Author:}

Dr. Kyawzaw Lin

Email: dr.kyawzawlin2015@gmail.com

This is an Open Access article distributed under the terms of the Creative Commons Attribution License (creativecommons.org/ licenses/by/3.0).

Received

Accepted

Published

August 19, 2017

October 15,2017

October 30, 2017

\begin{abstract}
Background: In the literature, the concurrence of pulmonary sarcoidosis in association with lupus pernio and peri-aortitis is extremely rare and has poor prognosis. The time lag between the diagnosis of sarcoidosis and aortitis varies from 6 months to 20 years. However, aortitis was diagnosed before sarcoidosis in a few cases. Case Report: We present a 49 -year-male with stage 4 pulmonary sarcoidosis and lupus pernio who presented with frequent exacerbation of asthma. Recent pulmonary function test revealed very severe mixed obstruction and restriction with predicted FEV $12 \%$ and DLCO 52\%. Chest X-ray showed diffuse bilateral lung fibrosis (stage 4 pulmonary sarcoidosis). CT scan (chest) showed widespread ground-glass attenuation with reticulo-nodular areas in both lungs and dilatation of thoracic aorta. Magnetic resonance angiography (MRA) revealed aortitis. Conclusion: Despite rarity, lupus pernio (LP) can be preceding sign of intra-thoracic involvement of sarcoidosis. In a few, peri-aortitis can be succeeding complication or concurrence of sarcoidosis. Corticosteroid or immunosuppressant is mainstay of treatment.
\end{abstract}

Keywords: Aortitis, Asthma, Dilatation, Lung, Respiratory Function Tests, Sarcoidosis.

\section{Introduction}

Although sarcoidosis was recognized more than 120 years ago, there are still many gray areas to explore. Sarcoidosis, a multisystem disease, is associated with serious cardiovascular complications such as myocarditis and granulomatous aortitis but cutaneous lesions are involved only in $25-35 \%$ of sarcoidosis [1]. Scarcely had concurrence of sarcoidosis with aortitis and cutaneous sarcoid particularly lupus pernio been only reported. However, the association has poor prognosis. Sarcoidosis usually preceded the diagnosis of aortitis by many years in general but in few, aortitis diagnosed before sarcoidosis is recognized as underlying cause.

\section{Case Report}

A 49-year-male with a decade of sarcoidosis (currently stage 4 pulmonary sarcoidosis) and lupus pernio (LP) presented with frequent exacerbation of asthma that is not well-improved with asthma medications. He was treated with cycles of tapering prednisone. His recent pulmonary function test revealed very severe mixed obstruction and restriction with predicted $\mathrm{FEV}_{1}: 21 \%$ and DLCO: $52 \%$ which was decreased by $37 \%$ and $14 \%$, respectively compared with those of 12 months ago. He denied fever, chest pain, abdominal pain, orthopnea, paroxysmal nocturnal dyspnea or palpitation. Physical examination was remarkable for violaceous nodules and plaques on face, nose, and chin compatible with lupus pernio [Fig.1, Panel a and b]. He was examined to have audible wheeze and tachypnea but able to speak full sentences with no accessory muscle involvement. Investigations showed normal white count, electrolytes including serum calcium, angiotensin converting enzyme (ACE) level and brain natriuretic peptide (BNP). Infection screening 
such as VDRL, HIV, chronic hepatitis B and C and vasculitis work up are unrevealing. Arterial blood gas was unremarkable except chronic hypercapnia. Electrocardiogram showed sinus tachycardia and chest X-ray showed diffuse bilateral lung fibrosis [Fig.2]. X-ray (both hands) showed no acute changes. Computed tomography $(\mathrm{CT})$ of the chest [Fig.3, coronal plane panel a and b] showed worsening widespread ground-glass attenuation with reticulo-nodular areas of bilateral lungs and irregular aortic thickening with dense adhesions suspicious for aortitis and dilatation of thoracic aorta $35.38 \mathrm{~mm}$ (normal: $<35 \mathrm{~mm}$ ). Subsequent magnetic resonance angiography (MRA) [Fig.4, panel $\mathbf{a}$ and $\mathbf{b}$ ] revealed irregular aortic thickening with dense adhesions which confirmed chronic peri-aortitis after ruling out other infectious aortitis including syphilitic, tuberculosis, etc. and noninfectious autoimmune vasculitis with serology. He was restarted with high dose prednisone $60 \mathrm{mg}$ daily in tapering dose and discharged home on $3^{\text {rd }}$ day with his baseline peak flow and recommended out-patient follow up in pulmonary clinic.

\section{Discussion}

Sarcoidosis is a chronic systemic granulomatous disease and usually presents with pulmonary manifestations in $90 \%$ of the cases and cutaneous involvement is estimated in $20-25 \%$ of cases. The most common dermatological presentation is
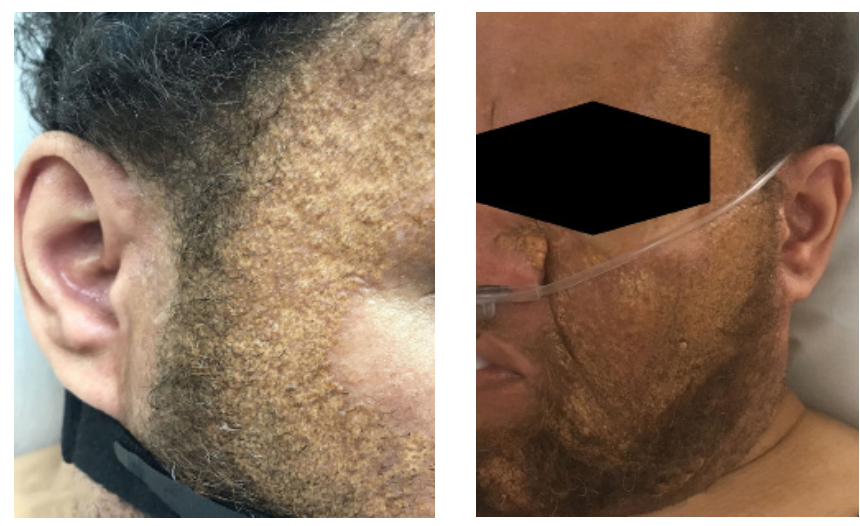

Fig.1: Panel a and b: violaceous nodules and plaques on face, nose, and chin (Lupus pernio).

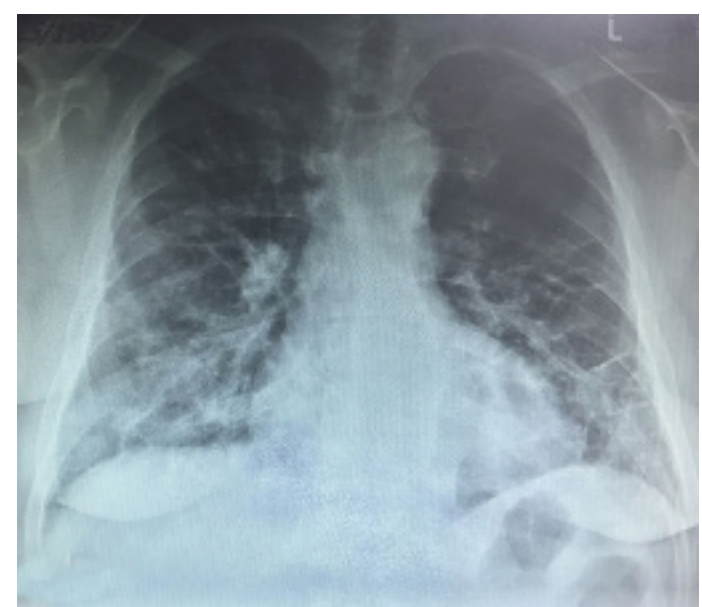

Fig.2: Chest X-ray showing diffuse interstitial pulmonary fibrosis.
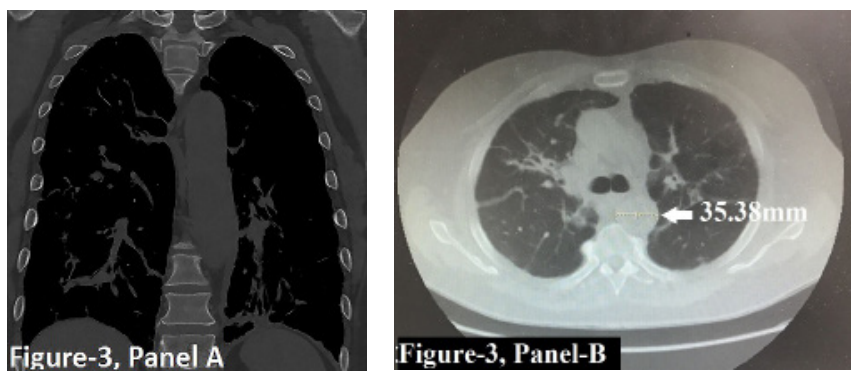

Fig.3: Panel a: CT of chest (coronal view) showed interstitial fibrosis. Panel b: CT of chest (cross-sectional view) showed dilatation of thoracic aorta $35.38 \mathrm{~mm}$ (normal: $<35 \mathrm{~mm}$ ) suspicious for aortitis.
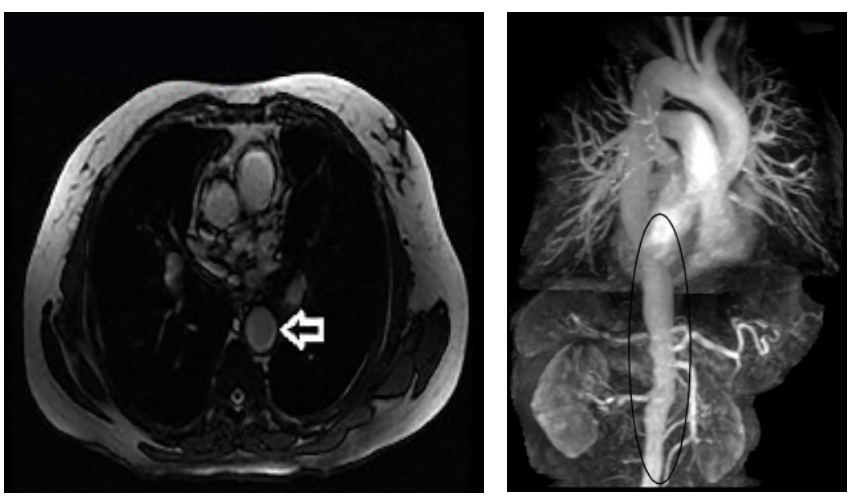

Fig.4: Panel a: MRA of abdominal aorta showed irregular aortic thickening with dense adhesions (arrow) consistent with aortitis and peri-aortitis. Panel b: MRA multi-planar reconstructed image of aorta showing irregular aortic thickening with dense adhesions confined to the abdominal aorta. 
erythema nodosum in sarcoidosis but the reverse is true for the lupus pernio (LP). LP is commonly distributed around forehead, nose, cheek, lips and ears and is characterized by indurated papular rash or plaques with varying colors. Definite diagnosis is obtained by biopsy and is refractory to treatment. More than $80 \%$ of sarcoidosis with LP is associated with intra-thoracic manifestation in female patients [2]. Lupus pernio is associated with chronic fibrotic interstitial lung diseases and resolution of those radiographic abnormalities is less in patients with lupus pernio [3].

In one clinical study done in 1976, 35 patients was found to have lupus pernio in a case series of 818 sarcoidosis patients confirmed with tissue biopsy and $74 \%$ of those patients with LP are associated with intra-thoracic involvement; 54\% with upper respiratory disease and another $54 \%$ with reticulo-endothelial involvement [4]. Lupus pernio is associated with advanced severe systemic involvement with chronic course. However, erythema nodosum runs acute benign course [5].

Similarly, aortitis or peri-aortitis is unrecognized and overlooked manifestation of sarcoidosis. Peri-aortitis can be a succeeding complication or concurrence of sarcoidosis [6]. Only $5-10 \%$ of cardiac sarcoidosis is symptomatic while the prevalence of $20-30 \%$ is found in autopsy. Whenever in doubt, high index of clinical suspicions was required to exclude aneurysm, aortic stenosis, and heart failure. Approximately $20 \%$ of patients present with irreversible clinical symptoms mainly such as pulmonary fibrosis while $12 \%$ of all stage 4 patients that present with complications such as pulmonary hypertension and aspergilloma require long-term oxygen therapy [7].

Only one case of sarcoidosis is associated with aortitis in a case report of 32 cases of noninfectious aortitis in a single tertiary center in Spain from January 2010 to December 2013 [8]. Another case of sarcoidosis presented with STEMI in setting of Valsalva sinus aneurysm and was resolved with thrombolytic but 6 months later, the underlying cause was aortitis in post-mortem examination [9]. A 39 years old female presented with erythema nodosum, circumscribed alopecia, and recurrent uveitis but 12 years later, she was diagnosed with progressive congestive heart failure in setting of aortic valve insufficiency. Sarcoidosis was later accidentally diagnosed from histological report from adjacent lymph nodes 17 years after initial presentation of sarcoidosis [10]. In 1994, Schapiro JM reported one case of a sarcoidosis patient with systemic symptoms without obvious vascular obstruction at the initial stage with pulseless Takayasu's arteritis [11]. 7-year-old boy with sarcoidosis since childhood presented with prolonged fever, iritis was accidentally found to be having abdominal aortic aneurysm, underwent uneventful repair in 1996 [12].

Inspite of poor predictive values, cardiac symptoms and electrocardiogram (EKG) are still used as screening tools while positron emission tomogram (PET) and cardiac magnetic resonance (CMR) are used as diagnostic purpose. Thus, in patients with cardiac symptoms with/without EKG abnormalities, imaging can raise diagnostic accuracy upto $20-30 \%$ while echocardiography with overall limited value can still be used as screening test [13]. Corticosteroid or immunosuppressant is mainstay of treatment. Surgical and endovascular intervention are intended for vascular occlusions and aneurysmal dilation.

\section{Conclusion}

Despite rarity of aortitis concurrent with sarcoidosis in the literature, lupus pernio is preceding sign of intra-thoracic association of sarcoidosis and has poor prognosis. Such concurrence can be manifested with life-threatening conditions such as aneurysm and heart failure. CMR is used not to diagnose but to confirm prognosis. Therefore, all sarcoidosis patients with cardiac manifestation with or without obvious EKG abnormalities might be a good candidate for CMR testing. 
Contributors: KL, SL: manuscript writing, patient management; ANL, TL, LG: manuscript editing, critical inputs into the manuscript, patient management. KL will act as guarantor. All authors approved the final version of the manuscript.

Funding: None; Competing interests: None stated.

\section{References}

1. Tong C, Zhang X, Dong J, He Y. Comparison of cutaneous sarcoidosis with systemic sarcoidosis: a retrospective analysis. International J Clinical Experimental Pathol. 2014; 7:372.

2. Yanardağ H, Pamuk ÖN, Karayel T. Cutaneous involvement in sarcoidosis: analysis of the features in 170 patients. Respiratory Medicine. 2003;97:978-982.

3. Spiteri MA, Matthey F, Gordon T, Carstairs LS, James DG. Lupus pernio: a clinico-radiological study of thirty-five cases. British Journal of Dermatology. 1985;112:315-322.

4. Neville E, Mills RG, James DG. Sarcoidosis of the upper respiratory tract and its relation to lupus pernio. Annals of the New York Academy of Sciences. 1976;278:416425.

5. Mañá J, Marcoval J, Graells J, Salazar A, Peyrí J, Pujol R. Cutaneous involvement in sarcoidosis: relationship to systemic disease. Archives of Dermatology. 1997;133:882-888.

6. Weiler V, Redtenbacher S, Bancher C, Fischer MB, Smolen JS. Concurrence of sarcoidosis and aortitis: case report and review of the literature. Annals of the Rheumatic Diseases. 2000;59:850-853.

7. Nardi A, Brillet PY, Letoumelin P, Girard F, Brauner M, Uzunhan Y, et al. Stage IV sarcoidosis: comparison of survival with the general population and causes of death. European Respiratory Journal. 2011;38:1368-1373.

8. Loricera J, Blanco R, Hernández JL, González-Gay MA. Non-infectious aortitis: a report of 32 cases from a single tertiary centre in a 4-year period and literature review. Clinical and Experimental Rheumatology. 2015;33(2 Suppl 89):S-19.

9. Marley WD, Booth K, Jeganathan R. Sarcoidosis or acute coronary syndrome: Easily distinguishable? Asian Cardiovascular and Thoracic Annals. 2016;24:54-56.

10. Maeda S, Murao S, Sugiyama T, Utaka I, Okamoto R. Generalized sarcoidosis with "sarcoid aortitis". Pathology International. 1983;33:183-188.

11. Schapiro JM, Shpitzer S, Pinkhas J, Sidi Y, Arber N. Sarcoidosis as the initial manifestation of Takayasu's arteritis. Journal of Medicine. 1993;25:121-128.

12. Gedalia A, Shetty AK, Ward K, Correa H, Venters CL, Loe WA. Abdominal aortic aneurysm associated with childhood sarcoidosis. The Journal of Rheumatology. 1996;23:757-759.

13. Kouranos V, Tzelepis GE, Rapti A, Mavrogeni S, Aggeli $\mathrm{K}$, Douskou $\mathrm{M}$, et al. Complementary role of CMR to conventional screening in the diagnosis and prognosis of cardiac sarcoidosis. JACC Cardiovascular Imaging. 2017 Mar 15. 